University of Wollongong

Research Online

Faculty of Law, Humanities and the Arts Papers (Archive)

Faculty of Arts, Social Sciences \& Humanities

$1-1-2015$

Looking beyond the brain: social neuroscience meets narrative practice

Daniel D. Hutto

University of Wollongong, ddhutto@uow.edu.au

Michael D. Kirchhoff

University of Wollongong, kirchhof@uow.edu.au

Follow this and additional works at: https://ro.uow.edu.au/lhapapers

Part of the Arts and Humanities Commons, and the Law Commons

Research Online is the open access institutional repository for the University of Wollongong. For further information contact the UOW Library: research-pubs@uow.edu.au 


\title{
Looking beyond the brain: social neuroscience meets narrative practice
}

\begin{abstract}
Folk psychological practices are arguably the basis for our articulate ability to understand why people act as they do. This paper considers how social neuroscience could contribute to an explanation of the neural basis of folk psychology by understanding its relevant neural firing and wiring as a product of enculturation. Such a view is motivated by the hypothesis that folk psychological competence is established through engagement with narrative practices that form a familiar part of the human niche. Our major aim is to establish that conceiving of social neuroscience in this wider context is a tenable and promising alternative to characterizing its job as understanding mentalizing as a wholly brain-based form of 'theory of mind' activity. To promote this change of view, it is shown that understanding folk psychology as a narrative practice can accommodate the known evidence from social neuroscience, developmental and cross-cultural psychology, and cognitive archaeology at least as adequately, if not better than its main rivals, modularist accounts of theory of mind.
\end{abstract}

\section{Keywords}

looking, meets, neuroscience, social, practice, brain, narrative, beyond

Disciplines

Arts and Humanities | Law

\section{Publication Details}

Hutto, D. D. \& Kirchhoff, M. D. (2015). Looking beyond the brain: social neuroscience meets narrative practice. Cognitive Systems Research, 34-35 5-17. 


\title{
Looking beyond the brain: \\ social neuroscience meets narrative practice
}

\author{
Daniel D. Hutto \\ Michael D. Kirchhoff
}

\begin{abstract}
Folk psychological practices are arguably the basis for our articulate ability to understand why people act as they do. This paper considers how social neuroscience could contribute to an explanation of the neural basis of folk psychology by understanding its relevant neural firing and wiring as a product of enculturation. Such a view is motivated by the hypothesis that folk psychological competence is established through engagement with narrative practices that form a familiar part of the human niche. Our major aim is to establish that conceiving of social neuroscience in this wider context is a tenable and promising alternative to characterizing its job as understanding mentalizing as a wholly brain-based form of 'theory of mind' activity. To promote this change of view, it is shown that understanding folk psychology as a narrative practice can accommodate the known evidence from social neuroscience, developmental and cross-cultural psychology, and cognitive archaeology at least as adequately, if not better than its main rivals, modularist accounts of theory of mind.
\end{abstract}




\title{
Looking Beyond the Brain: Social Neuroscience meets Narrative Practice
}

\author{
Daniel D. Hutto \\ Michael D. Kirchhoff
}

\begin{abstract}
"Gradually, we are discovering that we are social creatures with brains and minds that are part of larger organisms called families, communities and cultures ... to understand a person, we need to look beyond the individual"
\end{abstract}

- Cozolino 2014, p. xiii

It is beyond question that typically developing older human children and adults enjoy what might be neutrally called folk psychological (or FP) capacities. That is to say, there is clear and ample evidence that, at a certain point in development, ceteris paribus, human beings develop the capacity to make sense of actions done for reasons - whether the person in question is another or oneself. This is a structured capacity that involves making competent reference to a range of mental attitudes or predicates (e.g. belief, desire, hope, fear) respecting how such attitudes can inter-relate in complex ways.

Social neuroscience aims to contribute to our understanding of the neural bases of these capacities. Specifically, under the auspices of what might be called the 'what' strategy, social neuroscience seeks to characterize the function of a specific brain region. This goes significantly beyond the more basic 'where' strategy of identifying regions that are active during certain cognitive tasks (Anderson 2014, p. xvii-xviii). A number of obstacles block progress in delivering the relevant empirical answers in pursuing these strategies. Koster-Hale and Saxe (2013) acknowledge, for example, that neuroimaging techniques are limited in important respects: "they cannot decipher what is the input of a region, how that input is transformed, or where the output from that region is sent, during a ToM [theory of mind] task" (p. 156).

More concerning is the fact, as Koster-Hale and Saxe (2013) also admit, that, as things stand, social neuroscience is not even close to being able to answer fundamental questions about the ways we understand minds, including: "When and why do we (spontaneously) seek to understand another's thoughts?"; "How do we figure out the actual content of someone else's thoughts (i.e. what they are thinking) from specific cues?"; "How do we choose whether or not to incorporate others' thoughts into our own decisions and actions?"; and "Why do we care emotionally about others' thoughts and feelings?” (p. 156). 
Some hold out hope that technical developments in social neuroscience will, one day, put us in a position to address these issues: someday we may discovery more precisely what the brain is doing in making sense of minds, how it accomplishes this and "where in the brain mentalizing resides" (Mahy et al. 2014, p. 69, emphasis original). But there are philosophical reasons to doubt such a day will ever come. This will be so if understanding minds is not a matter of deploying a theory of mind. If we leave the theory of mind framework behind, the way forward for social neuroscience must be rethought. Conceiving of the nature of folk psychological competence and how it is acquired through enculturation in narrative practices gives social neuroscience a different role in helping us to explain how we understand minds.

\section{Theory of Mind in the Brain}

A staple assumption of much analytic philosophy of mind is that FP abilities just are theory of mind (or ToM) abilities. In using FP terms to understand minds we call on a set of rules or laws or principles that define how mental attitudes can inter-relate (Lewis 1970, 1978, Jackson 1998). Converting this basic idea into an explanatory proposal in cognitive science, a popular view is that ToM laws or principles are instantiated or contained in a species universal, biologically inherited module (which is variously characterized as a cognitive device, system, mechanism or computer). The common denominator in all ToM modularlist accounts is that ToM abilities are best explained by a cognitive architecture with a particular design and a dedicated, domain-specific function (BaronCohen 1995; Fodor 1983, 1995; Segal 1996; Scholl and Leslie 1999; Leslie et al. 2004).

The most important feature of modules, which separates them from other humdrum psychological mechanisms, is that they are cognitive through and through. They are: "symbol-manipulating devices which receive representations as inputs and manipulate them according to formally specifiable rules in order to generate representations (or actions) as outputs" (Samuels 2000, p. 18).

How seriously should we take the idea the modules literally embed a theory of mind? Carruthers (2011) complains that, "Gallagher and Hutto are mistaken in construing [modules] as purely thirdpersonal, or observer based ... [that Gallagher and Hutto] take the talk of 'theory theory' too strictly" (p. 231). Still, even if this correct, to deny that ToM modules are driven by rules and representations and do their work by manipulating concepts would be to make the idea of modules too weak to be of theoretical interest. Otherwise they will reduce to biologically inherited capacities that set us up for dealing with specific domains. The claim that modules are robustly cognitive is what puts the 'theory' in theory of mind modules. ${ }^{1}$

Fodor supplied the original formulation of how to understand other defining features of mental modules in his now classic, The Modularity of Mind (see Fodor 1983, part III). On his conception, modules: are informationally encapsulated (in that they are only receptive to certain kinds of inputs thus isolated from central cognitive processes); respond in mandatory, high speed ways; have low- 
level inputs and shallow outputs; instantiated in a fixed neural architecture; are prone to particular types of malfunction; and have a characteristic ontogenetic pace and sequencing.

Subsequent accounts of modules deviate from Fodor's specification of their basic features. For example, defenders of the massive modularity thesis - most prominently Carruthers (2011) abandon the idea that modules require information encapsulation. ${ }^{2}$ Even in their most stripped down form, modules are sometimes understood as nothing more than neurally realized, task-specific processing systems. Nevertheless, those proposing that ToM abilities are best explained by ToM modules also typically assume that such mindreading mechanisms are "an evolutionary adaption designed for the mental domain which is significantly innately channelled and early to emerge in development" (Carruthers 2011, p. 227).

This way of understanding the neural basis of ToM is alive and well in social neuroscience. For example, Samson and Michel (2013) tell us: "Making sense of other people's minds requires not only a set of processes that allow us to infer other people's mental states, but also long-term semantic knowledge about mental states that can be used to guide the inferential processes" (p. 171). ${ }^{3}$ That there might be ToM modules provides social neuroscience with a hard target and a clear agenda (see also Koster-Hale and Saxe's (2013) discussion of the 'strong hypothesis'). ${ }^{4}$ If such things exist, it is at least a credible project for neuroscience to go looking for the neural bases of this crucially important way of making sense of ourselves in human brains. It makes sense to speak of, and to search for ToM modules in individual brains, however distributed across such brains they may be. Against this set of background assumptions this sort of project of neural discovery, however difficult to conduct and achieve in practice, is a coherent theoretical activity.

There is a growing, empirically driven scepticism about the existence of dedicated ToM modules. On the upside, the modularist hypothesis makes strong predictions and hence has the merit (or explanatory virtue) of being highly falsifiable (see Mahy et al 2014, p. 70). On the downside, even if the existing evidence falls short of absolutely showing the modularist hypothesis to be false, when taken together, the empirical evidence provides no positive reason to believe it is true.

What would have to be the case, minimally, in order for a strong version of the modularist hypothesis to be true? As Mahy et al. (2014) observe: (1) a brain region, or network of brain regions, must be consistently activated in FP activity; (2) this pattern of activation needs to be present whenever there is FP activity, across all stages of development (Carruthers 2013) ${ }^{5}$; and (3) the region or network would need to be selectively deployed in FP (and only FP) activities (2014, p. 70). So far the empirical work provides no evidence that all three of these conditions are jointly satisfied. Indeed it points in the opposite direction.

A great deal of experimental work has been devoted to identifying, largely by means of correlative neuroimaging studies using subtraction methodology, the existence of a dedicated brain region or regions that are reliably involved in FP thinking (see Koster-Hale and Saxe (2013) for an overview). ${ }^{6}$ The main focus has been on two brain regions: (1) cortical midline structures (CMS) - including 
medial pre-frontal cortex (MPFC), adjacent rostral anterior cingulate cortex (rACC), and medial posterior parietal cortex (MPPC) - and (2) the bilateral temporal parietal junction (TPJ), both left (LTPJ) and right (R-TPJ). And, as Mahy et al. (2014) report, "the TPJ appears to be a [strong] candidate for a ToMM [theory of mind module]" (p. 70).

More specifically, functional magnetic resonance imaging (fMRI) experiments reveal that the right temporal-parietal junction (R-TPJ) is selectively enlisted for tasks requiring the interpretatively complex attribution of mental states. Saxe and Wexler (2005) discovered that R-TPJ activity is enhanced when the professed beliefs or desires of story protagonists conflicted with subjects' expectations about what such characters ought to believe or desire, based on background knowledge about them. Moreover, this region is not similarly recruited for other tasks that involve assessing other, more general, socially relevant facts about persons.

Saxe (2009) reports that the "fMRI literature suggests a division in the neural system involved in making social judgments about others, with one component (the R-TPJ) specifically recruited for the attribution of mental states, while a second component (the MPFC) is involved more generally in the consideration of the other person" (2009, p. 405). As Apperly (2011) also reports: "Altogether this series of studies suggest that regions of the R-TPJ, and to a lesser extent, L-TPJ and PC, respond in a selective way to written stimuli involving mental states ... These regions respond to beliefs that are known to be true or false, irrespective of whether these states need to be inferred or whether they are merely described" (2011, p. 66). This evidence has led a number of researchers to think that R-TPJ just might be a theory of mind module (see Apperly 2011; Koster-Hale and Saxe 2013; Mahy et al. 2014)

So far, so good, for modularists. This suggests that there may be some brain region that is consistently recruited in FP tasks, one that may even be necessary in a suitably strong sense for the completion of those tasks. But there is a fly in the ointment. For even if that proves to be so, it appears, that the relevant selectivity of this brain region comes late in the day, emerging "in the R-TPJ between ages 6 and 11 years" (Saxe 2009, p. 1206). Citing work by Kobayashi et al. (2007), in their systematic examination of the neural evidence of major theories of ToM acquisition, Mahy et al. (2014) also emphasize that "although 8 to 12 year-old children significantly engage TPJ bilaterally in ToM stories ... younger children do not always show this selective TPJ recruitment” (p. 70). The growing consensus is that the TPJ comes to be increasingly recruited and involved in FP modes of understanding minds. However, the evidence also indicates that there is no single, dedicated neural network at play in social cognition during all stages of development (Gweon et al. 2013).

In a bid to understand why this should be so and what might drive this process of increasing selectivity, Saxe and colleagues take seriously the "recent hint that middle childhood is a critical time for interactions between language and theory of mind" (Saxe 2009, p. 1207). Crucially, these new neuroscientific findings present a challenge for approaches to ToM development aiming to justify the existence of an innate and early-developed ToM module (see Saxe 2009, p. 1207). At the very least, 
these findings, if correct, present strong modularity theses - which assume that ToM modules are early-maturing - with a substantial problem (see Mahy et al. 2014, p. 71).

How does this conclusion fit with recent developmental evidence from experiments using violation of expectation and anticipatory looking paradigms, which show that children as young as 25 months (Southgate et al. 2007), 15 months (Onishi and Baillargeon 2005), and even 13 months (Surian et al. 2007) can pass language-free versions of false-belief tasks?

For some this evidence suggests that younger children must have, if not fully-fledged FP skills, at least a command of a concept of belief in place much earlier in development than demonstrated by their capacity to pass standard, verbally based, false belief tests (Baillargeon et al., 2010, Carruthers 2013). On rich, early ToM or 'mindreading' interpretations such findings are taken to establish that an infantile command of the concept belief is not dependent on mastery of the syntax, semantics or pragmatics of language. Combined with the neuroscientific findings cited above, the only credible way to sustain such ToM interpretations of the early infant data is to assume that there are two distinct ToM systems, one for implicit and another for explicit mindreading, where these involve different neurocognitive mechanisms. However, strong ToM interpretations of the infant data are highly controversial. They have been regularly challenged and a number of alternatives have been canvassed - ranging from wholly non-mentalizing proposals that regard the capacities in question to be nondomain specific or concerned only with behavior and not mental states to proposals that are domainspecific but understand the concern with mental states to be handled in a non-ToM manner (Heyes and Frith 2014, p. 1243091-3, for an overview of alternatives see Hutto et al. 2011). ${ }^{7}$ Whatever we decide is the best way to understand the basis of infantile social capacities, the neuroscientific evidence cited above puts pressure on the strong modularist assumption that there is a single ToM mechanism at play early on that remains fundamentally the same throughout all stages of development. Of course, it is possible to relax this condition and to argue that ToM modules are not early developing, after all, but only come on-line later in childhood.

How do modularist theories fare if we focus exclusively on the non-controversial cases of explicit FP abilities? It looks as if condition three - that the region or network would need to be selectively deployed in FP (and only FP) activity - is not met. Commenting on the 'lack of specificity' problem, Apperly reports "Legrand and Ruby (2009) review neuroimaging studies implicating ... regions of the "mindreading network" in mindreading, but also introspection, recalling information from memory, reasoning in general, and particularly inductive reasoning under conditions of vagueness and uncertainty" (2011, p. 62). Hence, even in adults, tasks that are not in need of ToM reasoning nevertheless evoke activity in the TPJ, thus violating the specificity criterion (see Mahy et al. 2014, p. 71). The evidence suggests that perhaps the general function of these regions is integrative, abstract reasoning. If this is correct, then it suggests that ToM is rooted in one of many domain-general functions of these regions (see Apperly 2011, p. 62). 
In addition, there is a concern that in over-emphasising the importance of the TPJ there is "a danger of excluding a yet broader set of neural regions that also serve important mindreading functions" (Apperly 2011, p. 70). Apperly nicely sums up the current state of social neuroscientific art with respect to its current picture of the basis of FP abilities:

The overall picture that emerges is surprisingly consistent. Mindreading recruits a complex network of functional and neural processes. Including among these are neural regions that appear highly selective for mindreading, but it seems clear that mindreading per se is a function of the network rather than of specific, specialized brain regions (2011, p. 59).

Although, as we argue below, Apperly's assessment does not go far enough it is, at least, a step in the right direction. It picks up on the fact that to successfully understand reasons in FP terms requires much more than merely knowing about the relevant mental state attitudes - for the attitudes in question always need to be appropriately situated against a larger narrative backdrop if we are to make sense of a person's reasons for action (Hutto 2008a, p. 7-8).

Before proceeding, it is important to be clear that our ambition is not to provide knockdown arguments against ToM modules in this paper. Instead we seek to promote an alternative way of thinking about the basis of FP - one that precludes the existence of ToM modules - and to show how such thinking might transform and re-direct the endeavors of social neuroscience in helping us to understand how we understand minds. ${ }^{8}$

\section{From Theory to Narrative Practice}

In light of the evidence just reviewed, let's go back to the drawing board and think again and afresh, about the cognitive basis of FP. Let's start by reviewing the assumption that FP reduces to having a ToM. Even though the proposed reduction is presented as being, near enough, an analytic truth there are compelling reasons to doubt it all the same (Hutto 2011b; Ratcliffe 2007). The Narrative Practice Hypothesis (NPH), for example, rejects the idea that FP reduces to or is embodied in a set of laws or principles but sees it rather as a competence fostered by engaging in socially supported story-telling activities (Hutto 2004, 2007, 2008a, 2008b, 2009).

The NPH accepts that at a certain point, assuming typical patterns of development, human beings begin to understand, explain and make sense of actions done for reasons. This is a structured capacity that sometimes involves making competent ascription of a range of mental attitudes or predicates (e.g. belief, desire, hope, fear), thus knowing how such attitudes can inter-relate in complex ways. Thus in at least some populations, adult humans do sometimes make sense of reasons by attributing propositional attitudes and contents. In those circumstances, mental state concepts and contents are attributed to others in systematic ways. 
The crucial question is, what is the basis of this mature human capacity? The fact that this kind of FP competence is structured does not entail that its structure derives from or requires the existence of a set of internalized rules stored in the brains of individuals (see Hutto 2012, pp. 50-53). The NPH offers an alternative to this familiar vision of the basis of FP. In the place of a set of ToM rules it puts a set of capacities that are shaped by narrative practice. The NPH holds that our facility with the forms and norms of FP is grounded in non-representational capacities that have been structured by social interactions and narratives.

For some it is intuitively difficult even to conceive of this capacity without assuming a set of internally represented rules. Apperly (2011), for example, says of this proposal: "I am sure that this is right to a significant degree, and can do a good deal to explain the basis of everyday social competence. Nonetheless, it seems equally clear that we cannot do away with the need for mindreading" (2011, p. 5, emphasis added). Yet Apperly supplies no developed argument to show why this should be the case. Overgaard and Michael (2015) make a better attempt. They argue that far from being an alternative to the ToM hypothesis, the NPH, if true, would only show that adult mindreading abilities are acquired through special narrative encounters. ${ }^{9}$ This will be so just in case the practical know-how such narrative encounters engender is:

also a matter of knowing that mental states are connected in such-and-such ways with other mental states and contextual factors. Indeed, if children did not abstract general principles from the various narratives they become acquainted with, it is difficult to see how they could learn to combine and modify them, etc., and thereby to bring them to bear upon novel situations. For they would be limited to memorizing specific narratives, which would hardly enable them to achieve understanding in real-life social situations that unavoidably differ significantly in the details. (Overgaard and Michael 2015, p. 167, emphases added).

Overgaard and Michael's (2015) second option is clearly a non-starter. The NPH does not propose that children are limited to making sense of reasons for actions by directly applying uniquely collected individual sets of remembered narratives. Instead the NPH proposes that children become familiar with the generalities - the constants and variables of the forms and norms of folk psychology through narratives. Their prior, explicit mastery of mental state concepts plays a part in the acquisition (and later exercise) of this FP competence.

Does it follow then that children are thereby imbibing and installing a set of ToM principles that become the internalized basis for their mature attribution of the attitudes? Must we take Overgaard and Michael's first option then? No. Even if it is accepted that FP competence can be formally described as a set of principles it does not follow that anything like such principles, in the form of a mentally represented set of laws, causally underpin or best explain our acquired FP competence (for detailed rebuttals of this non sequitur see Hutto 2008b, 2012; Strijbos \& De Bruin 2011). 
How is it possible to be sensitive to generalities without representing them as such? Telling the positive, non-rules and representation based account of the basis of FP would require explaining how qualitatively novel, intelligent activity can arise through long-term training without assuming that what is acquired in such training takes the form of instructional representations, programs, or algorithms (Araújo and Davids 2011, Hutto and Myin 2013, pp. 46-50). There is reason to think this can be achieved through the development of non-representational, enactive and embodied accounts of cognition. According to such approaches, humans develop and refine how they respond to ecologically available affordances through sustained embodied, engaged interactions. This enables greater contextsensitivity as skilled responding improves over time. A robust and fertile empirically inspired literature, drawing on the ecological dynamics framework, offers ways of understanding and explicating how dynamically embodied skills for social cognition can develop in constraint-led ways along the same lines that other structured skills, such as sporting abilities, are acquired. Importantly, this is an approach that shuns the intellectualist paradigm of classical cognitivism and its conceptual and contentful representationalist assumptions (Araújo 2009, Davids and Araújo 2010a, 2010b, Hutto, and Sánchez-García 2014). Enactivism seems well placed to provide an alternative to the familiar rules and representations account of how embodied know-how of the required sort is possible even without assuming the existence of ToM principles.

Assuming that such an account is explanatorily adequate the NPH can account for how we acquire our FP competence through participation in narrative practices and deploy it effectively without assuming the existence of a ToM. How does engaging with narratives, understood as cultural artifacts, make this possible? The NPH advances the view that we acquire FP competence through dealing with special kinds of narratives - folk psychological narratives - when appropriately supported by caregivers. Folk psychological narratives are a special sub-set of narratives - those that make mention of and show how mental states figure in the lives, history and larger projects of their owners. ${ }^{10}$ What they have in common with all narratives is that they depict a particular series of events in the lives of certain protagonists. Where they differ is that they detail the various psychological states and attitudes involved.

To properly understand the NPH, it is important to distinguish stories from narratives: A "'story" consists of all the events which are to be depicted ... 'Narrative' is the showing or telling of these events and the mode selected for that to take place" (Cobley 2014, p. 5). As the concrete renderings of stories, narratives are their particular and specific material manifestations. The same story can be conveyed by narrative means through a multitude of media including, indicatively, "mime, painting, stained glass windows, cinema, comics, news items, and conversations" (Cobley 2014, p. 213-214).

Narratives are thus special kinds of representations - but they are not primarily mental representations. They are artefacts - representational artefacts but also ordinary socio-cultural, public artefacts. They are literally stuff of "memoir, biography, autobiography, diaries, archival documents ... folk ballads ... and other art work" (Cobley 2014, p. 214). As complex linguistic representations - 
out-in-the-open items in our social landscape - folk psychological narratives can be anchors of joint attention in socio-cultural practices. The NPH goes further in assuming that public "tokens that have proper linguistic uses in relevant circumstances, hence play linguistic roles ... serve as the primary bearers of semantic content" (Haugeland 1990, p. 410). Of course, more work needs to be done to show how this can be the case, but there is no reason in principle to think that such an explication is impossible (Hutto and Satne 2015). Hence there is no a priori reason for ruling out the conceptual possibility of acquiring FP without acquiring a set of represented rules.

Building on this, the basic idea of the NPH is thus that "storytelling practices scaffold folk psychological reasoning, or reasoning about one's own and others' reasons for acting" (Herman 2013, p. 94). On the strong reading of the NPH, proposed here, the scaffolding in question does not simply build up something that was already there, in essence, in a weaker form. That is to say engaging with narratives does not simply put the icing on a pre-existing ToM cake; rather, it is through participating in narrative practices that such a cake is baked for the first time. Thus "it is FP and ToM that are facilitated by narrative rather than vice versa" (Cobley 2014, p. 227, see also Herman 2013 p. 296298). Importantly, what is acquired in this process is not an internalized set of rules - we do not acquire a ToM (see Hutto 2012, p. 50-53).

The upshot is that if it can be appropriately supported by non-representationalist accounts of basic cognition, the NPH offers a truly alternative vision of FP - one that does not see FP as rooted in a set of represented laws stored in the brain of individuals, but as a structured competence gained by engaging in narrative practices. Moreover, the exercise of our FP competence does not take the form of applying a set of rules but is a skilled narrative practice in which we make sense of intentional actions by producing and consuming reason explanations (Hutto 2008a).

How does the NPH hold up evidentially? There are a number of empirical findings that the NPH can accommodate at least as adequately as, if not better than, its main ToM competitors.

For example, the NPH "predicts that if cultures diverge in significant ways in the profile of their narrative practices, we can expect to find different local tendencies and proficiencies in the use of folk psychology" (Hutto 2008a, p. 188). Many studies in cross-cultural psychology reveal significant socio-cultural variation in FP and FP-related abilities (e.g. Avis and Harris 1991; Naito and Koyama 2006; Vinden 1996). ${ }^{11}$ Assessing a sea of cross-cultural data, Mayer and Träuble (2012) reach the cautious conclusion that "it is not clear whether it is justified to assume a universal onset of false belief understanding across cultures" (2012, p. 22). Plenty of cross-cultural variation has been found when comparing non-Western and Western societies with respect to FP-related understanding. The standard explanation for such differences is put down to the fact that the "early social experiences of children [in such societies] clearly differ from the experiences of children in Western cultures" (Mayer and Träuble 2012, p. 22).

Consider, for example, Mayer and Träuble's (2012) own new study of the onset of FP understanding in Samoan children. Like other cross-cultural researchers, they choose to look at this 
particular population precisely because "ethnographical work reports different practices of intersubjective understanding" (Mayer and Träuble 2012, p. 22, emphasis added). A plausible explanation of the differences in the timing of the emergence and application of FP-related abilities in this case focuses on how, and how frequently, mental states terms are used. Samoans do not often speak about mental states per se. This is because, as cultural anthropologists observe, "people there frequently assert that it is impossible or very difficult to know what others think or feel" (Robbins and Rumsey 2008). As a result it looks as if discursive practices are the critical ones. In tune with the NPH, many researchers assume that FP-related performance differences may well be connected to the fact that Samoan "children might be exposed to less mental state talk - at least to a specific kind of it" (Mayer and Träuble 2012, p. 23).

Other recent work even more directly examines the possibility that narrative practices per se may play an explanatory role in the onset and performances of FP-related abilities. Fiebich (2014), for example, looks at the role narrative practices may play in the development of FP. She too focuses on false belief tasks, discussing research that highlights delayed belief understanding in the populations of some Eastern cultures (Hong Kong, Japan) as compared with their Western (US, western Europe) counterparts (Doan and Wang 2010; Liu et al. 2008). Notably, she connects this with the fact that different kinds of narrative practices predominate in these societies: Westerners tend to favour socalled mentalistic narratives, whereas Eastern narratives put far less emphasis on states of mind of individuals and focus more on situational factors to explain behaviour and action. Thus:

Differences in narrative practices are present already in early mother-child conversations. Caretakers from Western cultures seem to be engaged primarily in mentalistic narrative practices, whereas caretakers from Asian cultures exhibit a preference for behavioral-contextual narrative practices. For example, when reading a picture book together with their child, European and American mothers have been found to refer to the mental states of the protagonist (e.g., "the bear is sad") rather than to the embodied aspects of those states (e.g., "the bear has tears on his face"), contrary to Chinese mothers (Fiebich 2014, p. 6). As Fiebich (2014) argues, given that differences in narrative practice appear to correlate with FP performance, it is possible that the former might, at least partially, explain the latter. ${ }^{12}$

Other cross-cultural evidence is also broadly supportive of the NPH. Wellman and Peterson (2013) found variations in the developmental sequence in which the component elements of FP competence are acquired. Being fully FP competent is a complex skill. It requires coming to grasp a range of different psychological attitudes, their special characteristics and how they relate to one another. Wellman and Peterson (2013) distinguish amongst this fundamental set of ways of understanding attitudes, defining them and labeling them as follows: that people can have diverse desires concerning the same object (Diverse Desires, DD); different beliefs about the same situation (Diverse Beliefs, DB); that something can be true while the person may not know that (Knowledge Access, KA); that 
something might be true while the person believes otherwise (False Belief, FB); and that someone can feel one way but display a different emotion (Hidden Emotion, HE) (2013, p. 57).

What is important is that the order and sequence by which children get to grip with these attitudes and how they inter-relate with other attitudes varies socio-culturally. Based on a series of studies involving over 500 preschool children in the USA, Canada, Australia and Germany, it was found that $80 \%$ of children exhibit a common pattern of acquisition, developing their FP-related understanding in a $\mathrm{DD}>\mathrm{DB}>\mathrm{KA}>\mathrm{FB}>\mathrm{HE}$ sequence.

However, it has been discovered that Chinese and Iranian preschoolers come by their FP understanding in a different order, acquiring KA competence prior to DB (Shahaeian, et al. 2001; Wellman et al, 2006, 2011). Once again the preferred explanation of these differences is tied to local "conversational-cultural preferences for emphasizing knowledge acquisition vs. belief differences" (Wellman and Peterson 2013, p. 58). What is important, Wellman and Peterson (2013) tell us, is that they "believe that sequence similarities from one culture to the next, coupled with cross-cultural differences like these, are especially important and revealing" (2013, p. 58). We agree. Certainly, added to the mix, these sorts of findings create a nice body of existing cross-cultural evidence that is compatible with the NPH and is consistent with possibility of explaining FP development and performance in terms of systematic variations in local narrative practices. It is worth investigating whether the conversational preferences manifest themselves in and are driven by the different narrative practices in different human populations around the globe.

The point of this review of cross-cultural findings is not to suggest that such data defeats the hypothesis that we might have species universal ToM modules. Modularists can surely also accommodate such findings. They might hold, for example, that ToM modules are parameterized such that their particular onset and roll out sequences are sensitive to - triggered and tuned by - the local, and in these cases socio-cultural, environments and their particular discursive practices. This could explain local, cultural variations even if the basic ToM modules are universal in the species.

But if the modularists' answer is to be convincing they will need to assure us that relevant kinds of discursive practices were in place at the relevant points in pre-history - namely, at the right point in time to have made a difference to the forging of the original ToM modules such that they would have special settings to be tuned to culturally variant patterns. The logic is simple: If discursive practices do play a critical role in the onset and sequencing of FP-related capacities in ontogeny, then discursive practices of the appropriate sort would have had to have been a normal feature of our ancestral environment at the right time in pre-history when species universal ToM modules were allegedly being formed and forged.

Were they? As argued in Hutto (2008a) a plausible story is that inherited ToM modules co-evolved with discursive practices rather late-in-the day. But the timing seems off. For the co-evolution hypothesis would seem to require that discursive practices of the relevant kind were in place prior to 100,000 b.p., before the last group photo of our common ancestors prior to their Diaspora out of 
Africa, if it is assumed that ToMs are universal in our species. This seems unlikely. For even if we assume that discursive practices of some kind were in place before then they would have been in their infancy at best. It is likely that only after a very long period after language-ready humans first appeared on the scene that language would be sophisticated enough to support the relevant discursive practices.

In this regard it is important to note that even avid modularists admit, "It is only around 50,000 years ago that fully modern theory of mind abilities evolve" (Mithen 2000, p. 496) and that "A theory of mind had in all likelihood evolved by 40,000 years ago, but ... before this time there is as yet no clear evidence for it" (Baron-Cohen 1999, p. 273). One way modularists might deal with these findings would be to argue that biological evolution operated in a quick and parallel way across the entire human species such that the ToMs that evolved all took the same form in each and every human population.

Fair enough. Still, an even easier way to understand the link between FP and linguistic abilities is to accept the NPH proposal that understands the emergence of FP practices to be matter of the development of socio-cultural narrative practices. For as Sugiyama observes, "the practice of storytelling is ancient ... [and] language, an obvious prerequisite for storytelling, is likely to have emerged by 50,000 ... although the oldest known narrative (The Epic of Gilgamesh) dates back only 5,000 years ago" $(2001$, p. 233$){ }^{13}$

In sum, philosophically speaking, the NPH is at least as theoretically tenable as its modularist rivals and there is nothing that we securely know about human pre-history or the development of FP across cultures that casts empirical doubt on the NPH's alternative proposal about the basis of FP.

\section{From Modules to Niches}

Suppose that FP competence does not reduce to having a ToM and therefore does not require a biologically inherited ToM module. How else, then, might we think about the ultimate origin of our FP capacity? The NPH is a special case of the Scaffolded Mind Hypothesis, which holds that "human cognitive capacities both depend on and have been transformed by environmental resources" (Sterelny 2010, p. 472), and vice versa (see Hutto 2008a, p. 242, Hutto 2009, 26-27).

Evolutionary psychologists and modularlists, such as Tooby and Cosmides (1995), think that humans are exclusively adapted to their ancestors' Pleistocene environments, with a "confederation of hundreds or thousands of functionally dedicated computers (often called modules) designed to solve adaptive problems" (1995, p. xiii). In stark contrast, those attracted to the scaffolded mind hypothesis think of socio-cultural practices as making possible certain important human cognitive capacities.

But why imagine human cognitive capacities were fully formed in, and are locked into, the Pleistocene? Dupré identifies the standard reasoning: 
Recent history, it is generally assumed, is far too short to produce significant chances in the human genome, so to explain why we have the genes we do we must resort to long tracts of time during which humans were developing their distinctive endowments $[\ldots]$ that distinguish them from their nearest relatives (2001, p. 21).

As Dupré further notes: "This is generally assumed to be the million or so years preceding modern recorded history, the late Stone Age or Pleistocene" (2001, p. 21). However, this narrow focus on genotypic evolution turns - as Menary has recently pointed out - on an understanding of "evolution as an asymmetrical relationship of selective pressures from environments to brains" (2014, p. 290). This assumption overlooks and downplays the powerful role that human activity - in the form of sociocultural practices - plays in constructing and modifying our environments in ways that are evolutionarily, ecologically, and cognitively consequential.

Take literacy, for instance. Our capacity for literacy cannot be explained by direct appeal to cognitive devices forged in the Pleistocene by natural selection. Rather, as Heyes \& Frith (2014) argue, "because literacy originated only 5 to 6 thousand years ago, these specialized neurocognitive mechanisms are thought to be products of cultural inheritance or cultural evolution" (2014, p. 1243091-1). And, in precisely the same vein and for similar reasons, they argue that "mind reading, like print reading or literacy, is culturally inherited" (2014, p. 1243091-1).

The niche construction paradigm provides an alternative means, other than modularism, for understanding how this could be so. It recognizes that organismic abilities and environmental features co-evolve and, thus, deeply influence one another. Niche construction is "the process whereby organisms, through their metabolism, their activities, and their choices, modify their own and/or each other's niches" (Odling-Smee et al. 2003, p. 419; see also Laland et al. 2000, p. 132). Organisms adapt to environmental pressures. But they also - as Odling-Smee and colleagues emphasize construct, alter, and modify their own niches. In the animal kingdom, the construction of niches figures centrally in the explanation of the adaptive complementarity of certain organisms and their environments. In such cases, there is a dynamic, reciprocal interaction between the two. Organisms drive environmental change and organism-modified environments shape organisms. These creations, in turn, influence and shape the animals that create them. Many animals create external structures that shape their environment. Some crickets construct elaborate horn-shaped burrows (Turner 2000), beavers build dams and spiders fashion webs.

Much niche construction also concerns "making cognitive tools and assembling other informational resources that support and scaffold intelligent action" (Sterelny 2010, p. 465). Especially, in this way: "Humans profoundly modify both the physical and the informational environment of the following generations" (Sterelny 2010, p. 470). It is precisely here that we find the driving idea behind the NPH. We create communal artifacts - stories with special properties. Such 
stories, by featuring in our shared storytelling practices, are the basis of our capacity to make sense of ourselves and others in terms of reasons.

Although the NPH fits within Sterelny's broad vision of scaffolded minds, it does not sit perfectly well with every aspect of his particular rendering of that idea. A possible tension exists between the NPH and Sterelny's official account. Whether this is a real tension turns on how one understands Sterelny's emphasis on information processing and transmission and his framing of scaffolding in terms of an informational environment and of information as being transmitted from one generation to the next.

On a weak, non-contentful reading of information this is consistent with the NPH but explanatorily opaque. It is not explanatorily revealing to think of niche construction as primarily a modification and transmission of information. No one would deny that niches are (in part at least) constructed via socio-cultural practices. Trivially changing practices involve changes to informational structures (understood in the weak sense). Even so, talk about the transmission of information as opposed to the replication of material practices fails to capture what is most important about what is preserved down the ages. Explaining apprenticeship in terms of the transmission of information as opposed to preservation of embodied practices is rather like asking a friend to buy you a certain configuration of atoms when what you really want is an espresso: both are strictly true, but the former is pragmatically unhelpful.

On the other hand, a strong reading of information transfer promotes the misleading conception that practices are not just describable in terms of sets of principles but that sets of principles are required for and form the very basis of practices. To talk of what is preserved down the ages in such terms implies an unwelcome conception that bodies of represented knowledge are being passed on through socio-cultural practices. Here Hutchins (2011) sounds an important warning cry, reminding us that: "Cultural practices include particular ways of seeing (or hearing, or feeling, or smelling, or tasting) the world. Cultural practices are not cultural models traditionally construed as disembodied mental representations of knowledge. Rather they are fully embodied skills" (2011, p. 441).

We concur. Indeed, this is so even if the NPH is right that the socio-cultural practices that matter in the case of FP are narrative practices that trade heavily in external representations. The point is that representation-involving narrative practices cannot be understood in exclusively representational terms. This is because, as discussed above, the narrative practices in question may be ultimately rooted in embodied skills that are not themselves based on the manipulation of representations.

Is the niche construction proposal scientifically sound? There is some controversy in the biological sciences over the more extravagant claims made on its behalf - those that regard it as requiring a serious overhaul of traditional selectionist thinking (see Laland et al. 2000). But in order to secure their fortunes the scaffolded mind hypothesis and the NPH need only rely on the notion of niche construction in a quite understated form. And this is good since even those skeptical of the stronger claims made by fans of niche construction theory admit that: 
Our disagreements are not about whether niche construction occurs (it clearly does) but about its implications for evolutionary theory: the advocate sees them as profound, whereas the skeptics see no reason why niche construction poses any problems for standard evolutionary theory, much less any reason for fundamental revision (Scott-Phillips et al., 2013, p. 1232).

All in all, niche construction theory even if only modestly construed affords a way a getting beyond modularist thinking about the basis of our FP abilities. ${ }^{14}$

\section{From Neural Networks to Patterned Practices}

The NPH, its links to niche construction theory and the supportive cross-cultural findings discussed in early sections of this paper all fit neatly with the new direction of social neuroscience. Today, we are told, "[u]nderstanding the effects of culture on the brain appears to be the newest trend in social cognitive neuroscience" (Roepstorff et al. 2010, p. 1051). Yet, when we drill down it is not culture per se - for talk of culture is too vague and broad - but specific patterned practices within cultures that are the appropriate focus of theoretical interest.

In arguing precisely this point, Roepstorff et al. (2010) highlight the fact that: "The concept of patterned practices in domain-specific material-discursive environments stresses that actors participate in particular ways of doing things in these joint activities, for example praying regularly in particular ways" (Roepstorff et al. 2010, p. 1052, emphasis added). Thus when so-called cross-cultural research is pitched at the right level of grain "[t]his leads to a specific approach: employ social patterns of practice instead of an abstract notion of culture to inform experimental design and participant recruitment" (Roepstorff et al. 2010, p. 1052).

In making a detailed case for this fine-tuning, of looking at specific practices within societies, Roepstorff et al. (2010) give attention to the specific practices of professional musicians noting:

$[\mathrm{O}]$ nly by bending the concept of culture to the level where it no longer becomes meaningful, can one talk of them as belonging to a 'specific' culture. Here, much more selectivity is afforded by the concept of patterned practices in the domain-specific material-discursive environments musicians are participants in: the concept points to embodied practices, the competent manipulation of material artifacts (i.e. instruments, notational systems), the highly specific social institutions or settings (i.e. orchestras, jam sessions) they take place in etc. (2010, p. 1053).

In short, new wave enculturated brain research needs to set its sight, not on whole cultures, because such a notion is too homogeneous, but on "the more specific, middle-range concept of patterned practice" (Roepstorff et al. 2010, p. 1051). This level of grain - the focus on local, patterned practices 
- is in perfect harmony with the NPH, and the scaffolded mind hypothesis construed under the auspices of niche construction theory in the way set out in the previous section. Echoing the core elements of the NPH, Roepstorff et al. (2010) tell us that the "patterning, of minds, brains and bodies, is certainly not a trivial process. Even for a young child, whose life is almost entirely about learning, and who is equipped with a brain conceivably at the peak of plasticity, it takes years of constant practice. This is not done in isolation, the child is not only immersed in an environment of structured practices but also usually surrounded by others, parents, siblings, care-takers who help and correct the process" (2010, p. 1052).

In a radical shift of thinking, the focus is no longer solely, or even primarily, on how brain-based cognition enables cultural practices to exist. To follow this lead one must give at least equal weight to the question of how the particulars of socio-cultural practices enable and sustain cognition.

In departing from the ToM framework, this way of thinking about the role of social neuroscience which situates neural activity within larger practices has important methodological consequences: "The programmatic research question now is: how do neural networks shape practices and how do these practices shape neural networks. This marks an important conceptual shift from systems to systems-inaction or processes ...- to the analysis of structured (patterned), maintained relations between embodied minds and their social, material and discursive "environments"” (Roepstorff et al. 2010, p. 1057).

To illustrate and support their claim, Roepstorff et al. (2010) make much of a clear and compelling study of the effects of enculturation on the brain, Näätänen et al.'s (1997) study on phoneme perception. ${ }^{15}$ This study reveals that some important cortical transformatory effects are facilitated by the brain's participation in certain patterned, socio-cultural practices.

Based on a mismatch negativity paradigm, where subjects are exposed to unattended sounds in certain rhythmic patterns, Näätänen et al. have established that the primary auditory cortex in the left hemisphere is highly sensitive to changes in predictable sound patterns (1997, p. 432). In the first set of these experiments (1997), Näätänen et al. utilized Finnish and Estonian language speakers because of a relatively small discrepancy between the two languages in terms of vowel structure, except that Estonian vowel space includes an additional vowel, /ô/, not found in Finnish. In the experiment, the speakers were also presented as deviants a prototype of this sound, along with vowels existing in both languages (/o/ and /ö/), and a non- prototypical vowel (located between/e/ and /ö/) (1997, p. 432; Roepstorff et al. 2010, p. 1053). The upshot was that Finnish speakers showed significantly higher mismatch negativity when exposed to prototypical vowels in their native language than when exposed to the Estonian vowel /õ/.

Despite making this staunch case Roepstorff et al. (2010) still talk of these enculturated patterns of practice as correlating with the neural and psychophysical patterns. But it is important not to fall back into old ways of thinking at this crucial juncture. Enactivist and ecological dynamic approaches to cognition provide a way around this problem by not treating the brain as an isolated explanatory unit. 
The brain is best conceived of as participating "in a system, along with eyes and face and hands and voice, and so on, that enactively anticipates and responds to its environment" (Gallagher et al. 2013). To adopt this enactivist understanding of the brain's role in cognition is to see it as embedded within a larger brain-body-world dynamic. As such cognition is not just realized in neural space but is a fundamentally interactive and relational phenomenon. To the extent that it makes sense to talk of correlations between 'internal' brain patterns and 'external' patterned practices - it is only because neural patterns come to have signature features, which might be described as 'neuronal signatures', because they are a smaller part of a larger pattern.

To accept the patterned practices approach to social neuroscience is, minimally, to admit that our capacities for understanding others and ourselves are not originally something interior. Through practice and participation we literally come to embody the ability to understand ourselves and others in immediate, spontaneous ways. We are able to activate and enact those capacities, deftly and with precision, in the right circumstances. But even if they are not originally interior some may be tempted think that these capacities subsequently become so. As they become 'second nature' might not they become installed within us, within individual brains?

After all, even the main proponents of the idea that brains are enculturated hold that: "A patterned practice approach assumes that regular, patterned activities shape the human mind and body through embodiment, and internalization. Vice versa, enacting practices shape and re-shape norms, processes, institutions, and forms of sociality. Culture gets under the skin and skull, if you will, and it is remade gradually through collective instances of actualization" (Roepstorff et al. 2010, p. 1052, emphases added).

They tell us that:

Participating in patterns of practice mediates between culture and innate human capacities.

Patterns of practice thus coordinate neural networks in action and facilitate learning and adaptation as a process extending from the individual brain to sociality and material-discursive environments (Roepstorff et al. 2010, p. 1054, emphases added).

Warning, here lie monsters! We must beware of treacherous talk of internalization; it is potentially deeply misleading. Mastering FP through narrative practices, for example, is not a matter of internalizing and redeploying a set of rules of folk psychology (any more than we learn the 'rules' for jazz improvisation) - rules that are somehow stored as integrated mental representations in a dedicated neural network. The real danger is that having come this far we will fall at the last hurdle and continue to think and work in the shadow of the old ToM picture, failing to free ourselves of its constraining assumptions - indeed, doing so, without even questioning them or noticing how questionable they become as we begin to look beyond the head, and beyond the individual as the true source and basis of our FP capacities. 
Nor, in making best use of the basic capacities with which Mother Nature has initially supplied us, and augmenting and altering them by engaging in and with socio-cultural practices, is this best understood as a process of merely 'extending' what is already 'innately' there in 'individual brains'. The notion of the extended mind has notoriously troublesome connotations (see Hutto et al 2014). Strictly speaking, brainbound cognition does not leak out into the world, nor does culture leak in. The process of enculturated cognition can be understood better without reliance on such limited metaphors.

Here it is equally important not to be misled by the scaffolding metaphor into thinking that the surrounding practices that allow us to activate and exercise our FP capacities on any given occasion somehow disappear after the FP building is built, once our FP competence is 'installed'. For in making sense of our selves and others we are always using our capacities in response to the scaffolded offerings of the wider socio-cultural environment.

\section{Conclusion}

Only when social neuroscience abandons the last vestiges of what we dubbed the 'standard approach' - only insofar as it fully moves out of the shadow of the three traditional assumptions that FP is grounded in ToMs and thus ToM modules with dedicated neural hardware - will it be true to say that it is "discovering that we are social creatures" (Cozolino 2014, p. xiii). There is a positive future for social neuroscience if it replaces its old concern of trying to discover inherited neural mechanisms that underpin FP and undertakes the task of understanding the role that enculturated neural patterns play in enabling and being enabled by the active construction of narrative niches and practices.

\section{References}

Anderson, M.L. 2014. After phrenology: Neural reuse and the interactive brain. Cambridge, MA: MIT Press.

Apperly, I. 2011. Mindreaders: The cognitive basis of "theory of mind". Hove and New York: Psychological Press.

Apperly, I. 2013. Can theory of mind grow up? Mindreading in adults, and its implications for the development and neuroscience of mindreading. Understanding other minds: perspectives from developmental social neuroscience. Baron-Cohen, S. Tager-Flusberg, H. and Lombardo, M. V. Oxford: Oxford University Press, 72-92.

Apperly, I. and Butterfill, S. 2009. Do humans have two systems to track beliefs and belief-like states? Psychological review, 116, 953-970.

Araújo, D. (ed) 2009. Ecological approaches to cognition in sport and exercise. International Journal of Sport Psychology. 40: 1-227. 
Araújo D., and Davids K. 2011. What exactly is acquired during skill acquisition? Journal of Consciousness Studies. 18(3-4): 7-23.

Avis, J., and Harris, P. L. 1991. Belief-desire reasoning among Baka children: Evidence for a universal conception of mind. Child Development, 62, pp. 460-467.

Baillargeon, R., Scott, R., and He, Z. 2010: False-belief understanding in infants, Trends in Cognitive Sciences, 14, 110-118.

Baron-Cohen S. 1995. Mindblindness: An essay on autism and theory of mind. Cambridge, MA: MIT Press

Baron-Cohen S. 1999. The evolution of theory of mind. In The Descent of Mind: Psychological perspectives on hominid evolution, ed. MC Corballis, SEG Lea. Oxford: Oxford University Press

Butterfill, S. and Apperly, I. 2013. How to construct a minimal theory of mind. Mind and Language. 28(5). 606-637.

Carruthers, P. 2011. The opacity of mind: An integrative theory of self-knowledge. Oxford: Oxford University Press.

Carruthers, P. 2013. Mindreading in infancy. Mind and Language. 28(2), pp. 141-172.

Cobley, P. 2014. Narrative. London: Routledge.

Currie, G. 2010. Narratives and narrators. Oxford, Oxford University Press.

Cozolino, L. 2014. The neuroscience of human relationships. $2^{\text {nd }}$ Edition. New York: Norton.

Davids, K and Araújo, D. 2010a. The concept of 'Organismic Asymmetry' in sport science. Journal of Science and Medicine in Sport. 13, 633-640

Davids, K and Araújo, D. 2010b. Perception of affordances in multi-scale dynamics as an alternative explanation for equivalence of analogical and inferential reasoning in animals and humans. Theory and Psychology, 20(1), 125-134.

Doan, S. N., \& Wang, Q. (2010). Maternal discussions of mental states and behaviors: relations to emotion situation knowledge in European American and immigrant Chinese children. Child Development, 81(5), 1490-1503.

Dupré, J. 2001. Human nature and the limits of science. Oxford: Oxford University Press.

Fenici, M. 2013. Social cognitive abilities in infancy: Is mindreading the best explanation?, Philosophical Psychology. DOI: 10.1080/09515089.2013.865096

Fiebich, A. 2014. Narratives, Culture, and Folk Psychology. Phenomenology and the Cognitive Science. DOI 10.1007/s11097-014-9378-7. pp. 1-15.

Fodor JA. 1983. The modularity of mind. Cambridge, MA: MIT Press

Fodor JA. 1995. A theory of the child's theory of mind. In Mental Simulation, ed. M Davies, T Stone. Oxford: Blackwell 
Fodor, J. A. 2001. Doing without What's within: Fiona Cowie's critique of nativism. Mind. 110(437), 99-148.

Gallagher, S., Hutto, D.D., Slaby J., and J. Cole. 2013. The Brain as Part of an Enactive System. Behavioral and Brain Sciences. 36(4), pp. 421-422.

Gweon, H., Dodell-Feder, D. Bedny, M. and Saxe, R. 2013. Theory of mind performance in children correlates with functional specialization in a brain region for thinking about thoughts. Child Development. 83, pp. 1853-1868.

Haugeland, J. 1990. The Intentionality All-Stars. Philosophical Perspectives. 4. 383-427.

Herman, D. 2013. Storytelling and the sciences of the mind. Cambridge, MA: MIT Press.

Heyes, C. M. and Frith, C. D. 2014. The cultural evolution of mind reading. Science, 244(6190), pp. 1243091-1-1243091-6: DOI: 10.1126/science.1243091

Hutto, D.D. 2004. The limits of spectatorial folk psychology. Mind and Language, 19, pp. 548-573.

Hutto, D.D. 2007. The narrative practice hypothesis: Origins and applications of folk psychology In Narrative and Understanding Persons. Royal Institute of Philosophy Supplement, 82, pp. 4368.

Hutto, D.D. 2008a. Folk psychological narratives: The sociocultural basis of understanding reasons. Cambridge, MA: MIT Press.

Hutto, D.D. 2008b. The narrative practice hypothesis: clarifications and consequences. Philosophical Explorations, 11, pp. 175-92.

Hutto, D.D. 2009. Folk psychology as narrative practice. Journal of Consciousness Studies, 16(6-8), pp. 9-39.

Hutto, D.D. 2011a. Elementary mind minding, enactivist-style. In Joint Attention: New Developments in Philosophy, Psychology, and Neuroscience. Seemann, A. (ed). Cambridge, MA: MIT Press, pp. 307-341.

Hutto, D.D. 2011b. Presumptuous naturalism: a cautionary tale. American Philosophical Quarterly. 48(2), pp. $129-145$.

Hutto, D.D. 2012. Exposing the background: deep and local. In Knowing without Thinking: The Background in Philosophy of Mind: Radman, Z. (ed). Basingstoke: Palgrave. 37-56.

Hutto, D.D. and Sánchez-García, R. 2014. Choking RECtified: embodied expertise beyond Dreyfus. Phenomenology and the Cognitive Sciences. DOI: 10.1007/s11097-014-9380-0.

Hutto, DD. and Satne, G. 2015. The Natural Origins of Content. Philosophia.

Hutto, D.D., Herschbach, M and Southgate, V. (Eds). 2011. Special Issue on Mindreading and Its Alternatives. Review of Philosophy and Psychology, 2(3).

Hutto, D.D., Kirchhoff, M.D. and Myin, E. 2014. Extensive enactivism: why keep it all in? Frontiers in Human Neuroscience. 8(706), pp. 1-11.

Hutchins, E. 2011. Enculturating the supersized mind. Philosophical Studies, 152, pp. 437-446. 
Jackson, F. 1998. From metaphysics to ethics: a defence of conceptual analysis. Oxford: Oxford University Press.

Kobayashi, C., Glover, G., and Temple, C. 2007. Children's and adults' neural bases of verbal and nonverbal 'theory of mind'. Neural Psychologia. 45, pp. 1522-1532.

Koster-Hale, J. and Saxe, R. 2013. Functional neuroimaging of theory of mind. In S. Baron-Cohen, H. T. Flusberg and M. V. Lombardo (ads.), Understanding other minds: Perspectives from developmental social neuroscience (pp. 132-163). Oxford, UK: Oxford University Press.

Laland, K. N., Odling-Smee, J., and Feldman, M. W. 2000. Niche construction, biological evolution and cultural change. Behavioral and Brain Sciences, 23, pp. 131-146.

Lewis D. 1970. How to define theoretical terms. Journal of Philosophy, 67, pp. 427-46

Lewis D. 1978. Psychophysical and theoretical identifications. Australasian Journal of Philosophy 50, pp. $249-58$

Leslie A, Friedman O, German TP. 2004. Core mechanisms in 'theory of mind'. Trends in Cognitive Sciences, 8, pp. 528-33

Liu, D., Wellman, H. M., Tardif, T., and Sabbagh, M. A. 2008. A meta-analysis of false-belief understanding across cultures and languages. Developmental Psychology, 44, pp. 523-531.

Mahy, C.E.V., Moses, L.J., Pfeifer, J.H. 2014. How and where: theory-of-mind in the brain. Developmental Cognitive Neuroscience, 9, pp. 68-81.

Mayer and Träuble. 2012. Synchrony in the onset of mental state understanding across cultures? A study among children in Samoa. International Journal of Behavioral Development. 37(1), pp. $21-28$

Menary, R. 2014. Neural plasticity, neuronal recycling and niche construction. Mind \& Language, 29(3), pp. 286-303.

Mithen, S. 2000. Paleoanthropological perspectives on the Theory of Mind. In Understanding Other Minds, S. Baron-Cohen, H. Tager-Flusberg and D. Cohen (eds.), 488-502. Oxford: Oxford University Press.

Näätänen, R., Lehtokoski, A., Lennes, M., Cheour, M., Huotilainen, M., Livonen, A., et al. 1997. Language-specific phoneme representations revealed by electric and magnetic brain responses. Nature, 385(30), pp. 432-434.

Naito, M., and Koyama, K. 2006. The development of false-belief understanding in Japanese children: Delay and differences? International Journal of Behavioral Development, 30, pp. 290-304.

Odling,-Smee, F. J., Laland, K. N., and Feldman, M. F. 2003. Niche Construction: The Neglected Process in Evolution. Monographs in Population Biology, 37. Princeton, NJ: Princeton University Press.

Onishi, K., and Baillargeon, R. 2005. Do 15-month-old infants understand false beliefs? Science, 308, pp. 255-258.

Overgaard, S. Michael, J. 2015. The interactive turn in social cognition research: A critique, Philosophical Psychology. 28(2), 160-183. 
Ratcliffe, M. 2007. Rethinking commonsense psychology: a critique of folk psychology, theory of mind and simulation. Basingstoke: Palgrave Macmillan.

Robbins, J., \& Rumsey, A. (Eds.). 2008. Introduction: cultural and linguistic anthropology and the opacity of other minds. Anthropological Quarterly, 81, 407-420.

Rockwell, T. 2005. Neither brain nor ghost: A nondualist alternative to the mind-brain identity theory. Cambridge, MA: MIT Press.

Roepstorff, A. Niewohner, J. and Beck, S. 2010. "Enculturating brains through patterned practices". Neural Networks, 23, pp. 1051-1059.

Samson, D., and Michel, C. 2013. Theory of mind: Insights from patients with acquired brain damage. In S. Baron-Cohen, H. T. Flusberg and M. V. Lombardo (ads.). Understanding other minds: Perspectives from developmental social neuroscience (pp. 164-177). Oxford, UK: Oxford University Press.

Samuels. R. 2000. Massively modular minds: Evolutionary psychology and cognitive architecture. In P. Carruthers (ed.), Evolution and the human mind (pp. 13-46). Cambridge: Cambridge University Press.

Saxe, R. 2009. Theory of mind (neural basis). Encyclopedia of Consciousness. W. P. Banks. Amsterdam. Elsevier and Academic Press: 401-409.

Saxe, R. and A. Wexler. 2005. Making sense of another mind: the Role of the right temporo-parietal junction. Neuropsychologia 43(10): 1391-1399.

Scott-Phillips, T.C., Laland, K.N. Shuker, D. M. Dickins, T.E. and West, S.A. 2013. The niche construction perspective: a critical appraisal. Evolution, 68 (5), pp. 1231-1243

Schilbach, L. Timmermans, B., Reddy, V., Costall, A. Bente, G, and Schlicht, T. 2013. Towards a second-person neuroscience. Behavioural and Brain Sciences. 36. 393-462.

Scholl BJ, Leslie A. 1999. Modularity, development and 'theory of mind'. Mind and Language 14: $131-53$

Segal G. 1996. The modularity of theory of mind. In Theories of Theories of Mind, ed. P Carruthers, P Smith. Cambridge: Cambridge University Press

Shahaeian, A., Peterson, C.C., Slaughter, V. and Wellman, H.M. 2001. Culture and the sequence of steps in theory of mind development. Developmental Psychology, 47(5). pp. 1239-1247.

Southgate, V., Senju, A., and Csibra, G. 2007. Action anticipation through attribution of false belief by 2-year-olds. Psychological Science, 18, pp. 587-592.

Scalise Sugiyama, M. 2001. Food, foragers, and folklore: The role of narrative in human subsistence. Evolution and Human Behavior, 22, pp. 221-40.

Slors, M. 2009. The narrative practice hypothesis and externalist theory theory: For compatibility, against collapse. Journal of Consciousness Studies. 16(6-8), 335-359.

Sterelny, K. 2010. Minds: extended or scaffolded? Phenomenological and the Cognitive Sciences, 9(4), pp. 465-481. 
Strijbos, D.W. \& De Bruin, L.C. 2011. Making folk psychology explicit: the relevance of Robert Brandom's Philosophy for the debate on social cognition. Philosophia, 40, 139-163.

Surian, L., Caldi, S., and Sperber, D. 2007. Attribution of beliefs by 13-month-old infants. Psychological Science, 18, pp. 580-586.

Tooby, J., and Cosmides, L. (1995). The psychological foundations of culture. In J. Barker, J. Tooby and L. Cosmides (eds.), The Adapted Mind: Evolutionary Psychology and the Generation of Culture. Oxford: Oxford University Press, pp. 19-136.

Turner, S. 2000. The extended organism: the physiology of animal-built structures. Cambridge, MA: Harvard University Press.

Wellman, H.M. 2011. Developing a theory of mind. In U. Goswami (Ed). The Blackwell Handbook of Childhood Cognitive Development. $2^{\text {nd }}$ Edition. New York: Blackwell. pp. 258-284.

Wellman, H.M., Fang, F., Liu, D., Zhu, L. and Liu, G. 2006. Scaling of theory-of-mind understandings in chinese children. Psychological Science, 17(12), pp. 1075-1081.

Wellman, H.M. and Peterson, C. 2013. Theory of mind, development and deafness. Understanding Other Minds: Perspectives from Developmental Social Neuroscience. Baron-Cohen, C. TagerFlusberg, H. and Lombardo, M. V. Oxford: Oxford University Press. pp. 51-71.

Vinden, P. G. 1996. Junin quechua children's understanding of mind. Child Development, 67, pp. 1707-1716. 


\section{Notes}

This is precisely why in first introducing the idea of modules, Fodor objects to Chomsky's talk of mental 'organs'. He holds that such a description fails to capture the fact that mental modules presuppose the existence of "innately cognized propositional contents" (Fodor 1983, p. 5) and "innate concepts" (Fodor 2001, p. 110).

This is necessary since FP involves isotropic, open-ended forms of reasoning in which potentially any and "every fact we know is in principle relevant to success... [hence such forms of reasoning] rely on emergent characteristics of our entire system of knowledge" (Rockwell 2005, p. 24).

Samson and Michel (2013) hold that the dominant view in social neuroscience is still that ToM knowledge is based in general rules or laws about the mind - a kind of folk theory. Thus, although they note that the tendency to construe FP as a theory has been questioned it nevertheless remains, as compared to alternatives, "more widely accepted" (p. 171).

According to Koster-Hale and Saxe (2013) a strong hypothesis in social neuroscience is that one or more of the brain regions involved in understanding minds "has the specific cognitive function of representing people's mental states and experiences- that is, of thinking about thoughts. Whenever we are thinking about thoughts, there are neurons in these regions firing. These neurons are gathered in spatial proximity (i.e. into a "region") because they have related computational properties that are distinct from the computation properties of neurons in the surrounding cortex" (Koster-Hale and Saxe 2013, p. 143-144).

Carruthers (2013) defends the view that there is a "domain-specific mechanism implicated in mindreading competence is functional early in infancy ... [which] provides infants with the concepts and core knowledge necessary to represent the mental states of other agents, of all basic types (including beliefs that are false and appearances that are misleading). While the operations of this system probably become more streamlined and efficient with age, its representational capacities do not alter in any fundamental way" (p. 142). This is, of course, consistent with it being the case that "it takes time for children to become efficient at using their ToM and furthermore, that their ToM development occurs alongside important changes in their executive function and language abilities" (Samson and Michel 2013, p. 172). 
Completing any mindreading task will typically require the exercise of non-

mindreading capacities as well. So what is known as a subtraction methodology is used to isolate the mindreading areas and the mindreading areas alone: "The typical approach to this problem is to have participants complete a second task that makes the same general perceptual and cognitive demands as the mindreading task, but does not involve mindreading. If the tasks are well matched, then subtracting the neural activity recorded in the control task should leave the pattern of activity uniquely associated with mindreading" (Apperly 2011, p. 59).

Hutto (2011b) defends the view that there are both empirical and theoretical reasons for thinking that non-verbal infants (and other animals) might be, at best expert mind minders and not bona fide mindreaders of any sort. They may be sensitive and appropriately responsive to others' bodily expressed psychological attitudes in ways that foster expectations, incidentally, about more complex propositional attitudes, including contentful beliefs. Tracking intentional attitudes that happen to correlate systemically with beliefs would explain how nonverbals could have expectations about another's propositional attitudes, even if they lack the concept of 'belief' as such and are incapable of representing belief contents. In support of that view Fenici (2013), writes: "These experiments only attest that infants selectively respond to others' (false) beliefs. This does not yet demonstrate that they are attributing representational sates. Moreover, tracking capacities alone doe not specify the nature of what is tracked. Even though infants were effectively responding to others' beliefs, they might still do so by tracking simpler properties or features that are co-extensive with them" (Fenici 2013, p. 2-3, see also Apperly \& Butterfill (2009), Apperly (2011), and Butterfill \& Apperly (2013).

See also Schilbach et al. (2013) for a proposal in a similar spirit. For a different take on this threat of collapse of the NPH to theory theory, see Slors (2009).

As Currie observes, "Narrative is a form of representation; one with special features and a special role in our individual and collective lives ... Narratives are ... exquisitely suited to the representation of motive and action" (Currie 2010, p. 219). 
We talk here of FP-related abilities because researchers in the field have come to recognize that FP abilities do not reduce to the passing of false belief tasks. Even so, the false belief test is widely used in this research because it is so portable and makes for convenient comparisons. Thus, as Mayer and Träuble (2012) acknowledge, researchers are aware that "ToM cannot be limited to false belief understanding, false belief tasks [remain] the most popular tool to assess the child's ToM" (p. 21).

There are some anomalies in the data. For example, the findings by Liu et al. (2008) reveal that children from Beijing exhibit patterns of false belief understanding more akin to those of Westerners. What might explain this difference in performance within the Chinese-speaking population given the sort of narratives that are generally favoured in the wider Chinese culture? In the best case for the NPH, on closer inspection, the local narrative practices in Beijing and Hong Kong might be discovered to diverge in relevant respects. Thus it might be found that mental states are referred to more often in the stories and explanations of behavior provided in Beijing than is the case in Hong Kong. Yet even if this does not prove to be the case there may be other relevant differences in these populations concerning the precise nature of the local narrative practices. Here it is important to note that "The development of explicit mind reading is not only predicted by the frequency with which mothers use mental state terms - such as "think," "want," and "happy"-in conversation with their children; it is also predicted by the frequency of mothers" "causal-explanatory" statements about the mind, specifying relations between situations, mental states, and observable behavior" (Heyes and Frith 2014 p. 1243091-4). Notably, lending some initial support to the second possibility, Wellman and Peterson (2013) report that there are known differences between Chinese children in Beijing and their Western peers - specifically, the order in which they grasp some components of their FP-competence is reversed. This suggests there may be differences in the way these population discuss mental states with children (p. 58). 25).

To make this case in full would require demonstrating how the niche construction perspective allows us to explain or explain away all the phenomena that ToM modules are supposed to explain (e.g. the poverty of the stimulus arguments about FP, the inherited capacities that allow make humans prone to FP, and so on). For a discussion 
of how these issues might be dealt with in a way that is friendly to the NPH, see Hutto (2008, p. 181-186).

15 They describe the work by Näätänen and colleagues as "Arguably one of the best and most influential studies of enculturated brains" (Roepstorff et al. 2010, p. 1053). 